\title{
Exploring the potential of high power impulse magnetron sputtering for growth of diamond- like carbon films
}

Kostas Sarakinos, A Braun, C Zilkens, S Mraz, J M Schneider, H Zoubos, and Patsalas

\section{Linköping University Post Print}

N.B.: When citing this work, cite the original article.

Original Publication:

Kostas Sarakinos, A Braun, C Zilkens, S Mraz, J M Schneider, H Zoubos, and Patsalas, Exploring the potential of high power impulse magnetron sputtering for growth of diamondlike carbon films, 2012, Surface \& Coatings Technology, (206), 10, 2706-2710.

http://dx.doi.org/10.1016/j.surfcoat.2011.11.032

Copyright: Elsevier http://www.elsevier.com/

Postprint available at: Linköping University Electronic Press

http://urn.kb.se/resolve?urn=urn:nbn:se:liu:diva-75279 


\section{Exploring the potential of high power impulse}

\section{magnetron sputtering for growth of diamond-like carbon}

\section{films}

K. Sarakinos ${ }^{1,3,{ }^{*}, \text { A. Braun }}{ }^{1}$, C. Zilkens ${ }^{1}$, S. Mráz ${ }^{1}$, J. M Schneider ${ }^{1}$, H. Zoubos ${ }^{2}$, and P. Patsalas ${ }^{2}$

${ }^{1}$ Materials Chemistry, RWTH Aachen University, Kopernikusstr. 10, 52074 Aachen, Germany

${ }^{2}$ Department of Materials Science and Engineering, University of loannina, 45110 Ioannina, Greece

${ }^{3}$ Plasma and Coatings Physics Division, University of Linköping, 58183 Linköping, Sweden.

\section{Abstract}

Amorphous carbon films are deposited employing high power impulse magnetron sputtering (HiPIMS) at pulsing frequencies of $250 \mathrm{~Hz}$ and $1 \mathrm{kHz}$. Films are also deposited by direct current magnetron sputtering (dcMS), for reference. In both HiPIMS and dcMS cases, unipolar pulsed negative bias voltages up to $150 \mathrm{~V}$ are applied to the substrate to tune the energy of the positively charged ions that bombard the growing film. Plasma analysis reveals that HiPIMS leads to generation of a larger number of ions with larger average energies, as compared 
to dcMS. At the same time, the plasma composition is not affected, with $\mathrm{Ar}^{+}$ions being the dominant ionized species at all deposition conditions. Analysis of the film properties shows that HiPIMS allows for growth of amorphous carbon films with $\mathrm{sp}^{3}$ bond fraction up to $45 \%$ and density up to $2.2 \mathrm{gcm}^{-3}$. The corresponding values achieved by dcMS are $30 \%$ and $2.05 \mathrm{gcm}^{-3}$, respectively. The larger fraction of $\mathrm{sp}^{3}$ bonds and mass density found in films grown by HiPIMS are explained in light of the more intense ion irradiation provided by the HiPIMS discharge as compared to the dcMS one.

*Corresponding author (e-mail address: kostas@ifm.liu.se) 


\section{Introduction}

Diamond-like carbon (DLC) is a metastable form of amorphous carbon containing a significant fraction of $\mathrm{sp}^{3}$-hybridized bonds [1] which give rise to high hardness, chemical inertness, and optical transparency [1]. Due to these attributes, DLC films are widely used for surface protection in cutting tools [2;3], magnetic storage discs [4], biomedical devices [5-6], antireflection coatings, and optical sensors [7]. Various mechanisms have been proposed in order to describe the formation of $\mathrm{sp}^{3}$ bonds in DLC films [1,8-11]; among them, the most accepted view for amorphous carbon films without significant hydrogen incorporation is that the $\mathrm{sp}^{3}$ formation is triggered by subsurface densification generated by subplantation of energetic deposited species [1, 9-11]. In plasma assisted physical vapor deposition (PVD) techniques, the energetic species are predominantly inert gas $\left(\mathrm{Ar}^{+}\right)$and target-based (e.g. $\left.\mathrm{C}^{+}\right)$ions, and their kinetic energy can be controlled by the use of substrate bias voltage. Owing to this fact, PVD techniques which are able to provide relatively large fluxes of ions at the substrate, such as filtered cathodic arc (FCA) and pulsed laser deposition (PLD), have been shown to allow for growth of DLC films with $\mathrm{sp}^{3}$ fractions larger than $80 \%$ [1]. The above mentioned highly ionized PVD techniques, although successful on a laboratorial scale, suffer from relatively low deposition rates (PLD) [12] and lateral inhomogeneity (PLD, FCA) [13,14]. This severely challenges their feasibility for industrial scale applications. On the other hand, magnetron sputtering (MS) based techniques are widely employed in industrial processes due to their conceptual simplicity and their ability to deposit uniform 
films with relatively high rates. However, magnetron sputtering plasmas (in dc, pulsed and rf configuration) are characterized by relatively low electron densities (in the order of $10^{15} \mathrm{~m}^{-3}$ ) and low degrees of ionization (in the order of a few \%) of both gas and sputtered species [15]. This, in the case of amorphous carbon films, has been shown to lead to the growth of films with $\mathrm{sp}^{3}$ bond fractions significantly lower than those achieved by FCA and PLD, e.g. up to $45 \%$ in rfMS [16].

It is therefore evident that there is need for a sputtering-based technique which is able to provide energetic bombardment conditions necessary for deposition of DLC films with $\mathrm{sp}^{3}$ contents achieved by state-of-the-art ionized PVD techniques. High power impulse magnetron sputtering (HiPIMS) is a newly developed ionized PVD technique [17] that could meet the requirements mentioned above. In HiPIMS, the power is applied to the target in short (tens to hundreds of $\mu \mathrm{s}$ ) unipolar pulses of low duty cycle $(<10 \%)$ and frequency $(<2 \mathrm{kHz})[18,19]$. Depending on the pulse parameters (i.e. duty cycle and frequency) power target densities during the pulse on-time (also referred to as peak target power density) from several hundreds of $\mathrm{Wcm}^{-2}$ up to several $\mathrm{kWcm}^{-2}$ can be achieved. At the same time, the average target power density is maintained at the level of several $\mathrm{Wcm}^{-2}$ which is typical for conventional magnetron sputtering processes (e.g. dcMS) $[18,19]$. The high values of peak target power density result in generation of plasmas with densities between $10^{17}$ and $10^{19} \mathrm{~m}^{-3}$ [20] much higher than the typical values observed in dcMS plasmas in the range $10^{14}$ to $10^{16} \mathrm{~m}^{-3}$ [15]. The 
high plasma densities, in turn, have as a consequence high degrees of ionization of both gas and sputtered species (peak values up to $90 \%$ for $\mathrm{Ti}$ [21]) and peak substrate ion current densities up to three orders of magnitude higher than those obtained in dcMS processes [17]. These plasma conditions have been shown to enable deposition of ultra-smooth and dense films [22,23], allow for control over the film microstructure [24,25] and phase composition [26-28], and lead to films with superior mechanical [29] optical [28], and electrical properties [30,31]. As far as it concerns DLC, Bugaev et al. [32] and DeKoven et al. [33] have reported results on growth of amorphous carbon films by HiPIMS which indicated $\mathrm{sp}^{3}$ fractions up to $45 \%$ as estimated by Raman spectroscopy measurements. However, there is so far in the literature no attempt to define deposition conditions during HiPIMS that would allow for growth of high $\mathrm{sp}^{3}$ content DLC films comparable to those obtained by state-of-the-art ionized PVD techniques. Towards realizing this goal, in the present study, we grow amorphous carbon films for a variety of conditions in the HiPIMS parameter space and employ analytical tools to elucidate the process-plasma relationships during film growth. This information is utilized to understand the effect of the process parameters on the film bonding properties and explore the feasibility of HiPIMS for the deposition of DLC films.

\section{Experimental procedure}

Amorphous carbon films were deposited on 2 inch p-type (B doped) Si (100) substrates (resistivity 1-20 $\Omega \mathrm{cm}$ ) by HiPIMS and dcMS, for reference. The 
substrates were placed on a stainless-steel holder water-cooled at room temperature. Depositions were performed from a $90 \mathrm{~mm}$ in diameter and $3 \mathrm{~mm}$ in thickness graphite $(99.995 \%$ purity) target operated at a constant average power of $60 \mathrm{~W}$ in an $\mathrm{Ar}$ atmosphere with a pressure of $1.8 \mathrm{~Pa}$ at $70 \mathrm{~mm}$ target-tosubstrate distance. The magnetic field strength in the center of the target surface was $\sim 550$ G. For the HiPIMS experiments, unipolar power pulses were applied to the target using a MELEC 1000A pulsing unit fed by an ADL dc generator. The same experimental arrangement was also used for the dcMS depositions. The time-dependent current and voltage during the HiPIMS process were measured using a LEM-205-S current and ELDITEST GE 8115 voltage transducers, respectively, and monitored in a Tektronik TDS 2014B digital oscilloscope. It is known that in HiPIMS and for a given average target power and working pressure, the choice of the pulse duty cycle and frequency affects the value of the peak target power (or current) [19]. This value, in turn, has implications on the plasma density and ionization [19]. In order to provide the growing film with different ion fluxes, unipolar pulses with a width of $50 \mu$ s and frequencies (f) in the range between $250 \mathrm{~Hz}$ and $1 \mathrm{kHz}$, corresponding to duty cycles in the range 1.25 to $5 \%$, were used. Furthermore, the energy of the ionized species impinging onto the substrate was varied by applying negative unipolar pulsed (pulse width and repetition frequency $5 \mu$ s and $100 \mathrm{kHz}$, respectively) bias voltages up to 150 $\mathrm{V}$ using an ENI generator. 
The effect of the process parameters on the plasma chemistry and energetics was investigated by means of energy-resolved mass spectrometry. The massenergy analyzer PPM 422 (Pfeiffer Vacuum) was employed for the measurements. The distance between the target and the grounded mass-energy analyzer sampling orifice was $70 \mathrm{~mm}$ and the orifice diameter was $100 \mu \mathrm{m}$. Information regarding the ion nature was obtained by performing mass-to-charge measurements at the most probable energy of the dominant ions $\left(\mathrm{Ar}^{+}\right)$ corresponding to the plasma potential. Subsequently, ion energy distribution functions (IEDFs) of the ions identified in the plasma were measured to quantify their energy. Furthermore, the IEDFs were integrated over the scanned energy range and the ratios of the individual species' integrated intensities to the total integrated intensity of all IEDFs were calculated. These ratios were in turn used to unravel relative changes of the plasma composition as a function of the deposition conditions.

The film density and thickness were determined by means of X-ray reflectometry $(\mathrm{XRR})$. The XRR measurements were performed in a Bruker D-8 instrument equipped with a special reflectivity sample stage and a Goebel mirror. The XRR data have been analyzed using the procedures described in Ref. [34]. The film thickness (obtained by XRR) was divided by the deposition time to calculate film deposition rate. The latter quantity was found to be about $\sim 1,5 \mathrm{~nm} / \mathrm{min}$ for all deposition conditions. Auger Electron Spectroscopy (AES) was employed to determine the fraction of $\mathrm{sp}^{3}$ bonds in the films. The AES measurements were 
performed in an ultra-high vacuum $\left(<5 \times 10^{-8} \mathrm{~Pa}\right)$ system by $\mathrm{PHI}$ using a $1 \mathrm{keV}$ primary electron beam for excitation. The backscattered electrons and the emitted Auger electrons were selected using a cylindrical mirror analyzer (CMA$\mathrm{PHI}$ ) and detected with a channeltron. The energy resolution of that analyzer had a $\triangle E / E$ ratio of $0.6 \%$. The $\mathrm{sp}^{3}$ content of the films have been determined by AES from the broadening of the $\mathrm{C}_{\mathrm{KVV}}$ (or equivalent $\mathrm{C}_{\mathrm{KLL}}$ ) peak [35-37].

\section{Results and discussion}

\subsection{Plasma properties}

Mass-to-charge scans at constant energy (not presented here) revealed the presence of Ar-, C- and $\mathrm{H}_{2} \mathrm{O}$-related ionized species at all deposition conditions. The IEDFs recorded during dcMS and HiPIMS (frequency $\mathrm{f}=250 \mathrm{~Hz}$ ) for $\mathrm{Ar}^{+}, \mathrm{C}^{+}$ and $\mathrm{H}_{2} \mathrm{O}^{+}$species are plotted in Fig. 1 (a), (b) and (c), respectively. It can be seen that dcMS gives rise to IEDFs with a most probable energy of $\sim 1 \mathrm{eV}$ and width of several $\mathrm{eV}$. Irrespective of the species probed, the implementation of HiPIMS does not affect significantly the position of the most probable energy in the IEDFs whereas it leads to high energy tails up to $\sim 50 \mathrm{eV}$. Moreover, the maximum intensity of the IEDFs recorded for the HiPIMS process is larger by a factor of $\sim 10$ than that obtained for dcMS. The same qualitative trends (i.e. larger intensity and high energy tails) are also observed when comparing the IEDFs recorded for the HiPIMS process with a frequency $\mathrm{f}=1 \mathrm{kHz}$ (not shown in Fig. 1) to dcMS. The relative integrated intensities of the IEDFs for various species for dcMS and HiPIMS at pulsing frequencies of $1 \mathrm{kHz}$ and $250 \mathrm{~Hz}$ are plotted in Fig. 
2 (a), (b) and (c), respectively. It is seen that in all discharges, the relative integrated intensity of $\mathrm{Ar}^{+}$IEDF is dominant with values ranging from 85 to $90 \%$. The remaining fraction of 10 to $15 \%$ corresponds to $\mathrm{C}^{+}, \mathrm{Ar}^{2+}, \mathrm{Ar}_{2}{ }^{+}, \mathrm{H}_{2} \mathrm{O}^{+}$and $\mathrm{H}_{3} \mathrm{O}^{+}$ions. The values of the relative integrated intensities do not undergo significant changes among the various deposition conditions. On the contrary, the deposition conditions have implications for the total integrated intensity. The latter increases from $\sim 9 \times 10^{5}$ counts in dcMS to $\sim 2.3 \times 10^{6}$ counts in HiPIMS (pulsing frequency $\mathrm{f}=1 \mathrm{kHz}$ ) as shown in Fig. 2 (a) and (b), respectively. The decrease of the pulsing frequency to $250 \mathrm{~Hz}$ results in a further increase in the total integrated intensity to $\sim 3 \times 10^{6}$ counts (Fig. 2 (c)). This is accompanied by an increase in the peak target power from 1.5 to $9.5 \mathrm{~kW}$. It is worth noting that $\mathrm{C}^{+}$ ions constitute $\sim 1 \%$ of the total integrated intensity at all deposition conditions. This value is considerably lower than the values of $\sim 50 \%$ or larger measured for other Ar-metal HiPIMS discharges [19]. This fact implies that HiPIMS is not as efficient in ionizing $C$ which is in qualitative agreement with experimental reports [33] and model predictions $[38,39]$. This behavior can be explained by the relatively high ionization potential and the relatively low electron impact ionization cross section of C compared to most metals [40]. Thus, at the conditions employed in the present study, the implementation of HiPIMS for sputtering a C target does not lead to an increase of the fraction of ionized sputtered species in the deposition flux compared to dcMS. The difference between the two techniques lies on the fact that HiPIMS allows for a larger total ion flux (mainly consisting of $\mathrm{Ar}^{+}$ions) of larger average energy as manifested by the larger 
integrated intensities and broader IEDFs in Fig. 2 and 1, respectively. Furthermore, the increase of the peak target power (by decreasing the pulsing frequency) enables one to maximize the plasma ionization degree and thus the total ion flux towards the growing film.

\subsection{Film properties}

The effect of the negative bias potential $\left(V_{b}\right)$ applied to the substrate on the $\mathrm{sp}^{3}$ fraction in the a-C films deposited in the current study is presented in Fig. 3. It is seen that in the case of HiPIMS deposited films, an increase of $V_{b}$ up to a value

of $50 \mathrm{~V}$ results in an increase of the $\mathrm{sp}^{3}$ content in the films followed by a decrease at larger $V_{b}$ values. This trend is in qualitative agreement with results obtained by other PVD techniques, such as PLD [41], FCA [42] and rfMS [16] which are reproduced for reference in Fig. 3 . In the case of dcMS grown films, a continuous increase of the $\mathrm{sp}^{3}$ content up to $\mathrm{a} \mathrm{V}_{\mathrm{b}}$ value of $90 \mathrm{~V}$ is observed. One reason for the formation of $\mathrm{sp}^{3}$ bonds in amorphous carbon films is the densification caused by subplantation of energetic species [1]. The mass density of the a-C films grown by dcMS and HiPIMS as a function of $V_{b}$ is plotted in Fig. 4 along with published results from PLD grown films [12]. The film density initially increases with increasing $V_{b}$ followed by a decrease. In addition, the maximum value achieved by HiPIMS $\left(\sim 2.2 \mathrm{gcm}^{-3}\right)$ is larger than the corresponding dcMS value $\left(\sim 2.05 \mathrm{gcm}^{-3}\right)$ and considerably smaller than that in PLD (larger than 3.0 $\left.\mathrm{gcm}^{-3}\right)$ [12]. It is therefore evident from the results presented in Figs. 3 and 4 that the evolution of the fraction of $\mathrm{sp}^{3}$ bonds in our films is consistent with the 
predictions of the densification model [1]. According to this model the initial increase in the $\mathrm{sp}^{3}$ content is a result of the subplantation-induced densification, while the decrease at larger $V_{b}$ values is due to relaxation caused by the excess of energy provided to the growing film [9]. Furthermore, the differences in the changes of the $\mathrm{sp}^{3}$ content as a function of the substrate bias voltage between HiPIMS and dcMS grown films are consistent with the IEDFs presented in Fig. 1. The broader IEDFs recorded for the HiPIMS discharges imply, on average, larger ion energies. Thus a smaller bias potential is required to provide ions with necessary energy for subplanation and $\mathrm{sp}^{3}$ formation. Moreover, the maximum $\mathrm{sp}^{3}$ bond fraction achieved by HiPIMS ( 45 at- $\%$ for a pulsing frequency of 250 $\mathrm{Hz}$ ) is larger than that obtained by dcMS ( 30 at-\%). This can be explained by the larger total ion flux available during HiPIMS growth as manifested by the larger total integrated ion intensity in Fig. 2 . It is also seen that the maximum $\mathrm{sp}^{3}$ content obtained by HiPIMS is comparable to the values reported for rfMS, but significantly smaller than those delivered by FCA and PLD. The latter is consistent with the notion that FCA and PLD are PVD techniques able to generate large fluxes of ionized film forming species. Apart from the densification, the $\mathrm{sp}^{3}$ content in a-C films is also known to be promoted by $\mathrm{H}$ incorporation [43]. In Fig. 5, published density values for hydrogenated and nonhydrogenated a-C films deposited using a variety of physical and chemical vapor deposition techniques $[12,16,43-47]$ are plotted as function of the $\mathrm{sp}^{3}$ content. For relatively low $\mathrm{sp}^{3}$ bond fractions in the order of $20 \%$, our data follow the line that corresponds to the non-hydrogenated $\mathrm{a}-\mathrm{C}$ films. As the $\mathrm{sp}^{3}$ content 
increases, the mass density deviates towards the hydrogenated a-C line indicating the incorporation of $\mathrm{H}$ in our films. This is consistent with the fact that $\mathrm{H}_{2} \mathrm{O}$-related species have been detected in both dcMS and HiPIMS plasmas (Figs. 1 and 2). This indicates that the cleanliness of the vacuum chamber is crucial because in HiPIMS a higher amount of activated radicals and atoms can be produced, compared to the conventional dcMS; such radicals may easily be incorporated into the film and affect its properties [8]. Thus the increase of the $\mathrm{sp}^{3}$ bond fraction as a function of the substrate bias voltage (i.e. ion energy) observed in Fig. 3 can also be attributed to the increase of the $\mathrm{H}$ incorporation. Despite the latter, the implementation of HiPIMS results in film densification.

\section{Summary}

Amorphous carbon films were grown employing HiPIMS and dcMS, for reference. Plasma analysis showed that the implementation of HiPIMS and for the conditions (pulse width, power and frequency) employed in the present study did not affect the relative composition of the ionic population, i.e. $\mathrm{Ar}^{+}$are the dominant ionized species in all discharges. This can be understood by the considering the low ionization probability of $\mathrm{C}$ as compared to $\mathrm{Ar}$ atoms. However, the HiPIMS discharges were found to exhibit a larger number of ions with larger average energies, as compared to dcMS. This causes the growth of

films with $50 \%$ larger $\mathrm{sp}^{3}$ bond content and $7 \%$ larger mass density as compared to dcMS. Comparison of the mass density and $\mathrm{sp}^{3}$ bond fractions in our films with results reported in the literature indicate incorporation of $\mathrm{H}$ during growth. Our 
results demonstrate that the larger ion fluxes and energies generated during

HiPIMS enable densification of amorphous carbon films with larger $\mathrm{sp}^{3}$ bond content as compared to films grown by dcMS.

\section{Acknowledgements}

KS should like to acknowledge the financial support by the Swedish Research Council (VR) through the contract 623-2009-7348. JMS and SM gratefully acknowledge funding from the Deutsche Forschungsgemeinschaft (DFG) within the Collaborative Research Center SFB-TR 87.

\section{References.}

[1] J. Robertson, Mater. Sci. Eng. R37 (2002) 129.

[2] M. Hakovirta, Diam. Relat. Mater. 5 (1996) 186.

[3] W. Tillmann, E. Vogli, F. Hoffmann Surf. Coat. Technol. 204 (2009) 1040.

[4] A. C. Ferrari, Surf. Coat. Technol.180-181 (2004) 190.

[5] M. Stüber, L. Niederberger, F. Danneil, H. Leiste, S. Ulrich, A. Welle, M. Marin, H. Fischer, Adv. Eng. Mater. 9 (2007) 1114.

[6] A. Grill, Diam. Relat. Mater. 12 (2003) 166.

[7] N. T. Panagiotopoulos, P. Patsalas, C. Prouskas, G. P. Dimitrakopulos, P.

Komninou, T. Karakostas, A. P. Tighe, E. Lidorikis, ACS Applied Materials and Interfaces 2 (2010) 3052.

[8] S. Neuville, A. Matthews, Thin Solid Films 515 (2007) 6619.

[9] J. Robertson, Diam. Relat. Mater. 2 (1993) 984. 
[10] Y. Lifshitz, G. D. Lempert, and E. Grossman, Phys. Rev. Lett. 72 (1994) 2753.

[11] C.A. Davis, G.A.J. Amaratunga, K.M. Knowles, Phys. Rev. Lett. 80 (1998) 3280.

[12] P. Patsalas, S. Kaziannis, C. Kosmidis, D. Papadimitriou, G. Abadias, G.A. Evangelakis, J. Appl. Phys. 101 (2007) 124903.

[13] R. Eason (Ed.), Pulsed Laser Deposition of Thin Films: Applications-Led Growth of Functional Materials, Wiley 2006.

[14] A. Anders, Cathodic Arcs: From Fractal Spots to Energetic Condensation, Springer 2010.

[15] C. Christou, Z.H. Barber, J. Vac. Sci. Technol. A 18 (2000) 2897.

[16] S. Logothetidis, M. Gioti, P. Patsalas, C. Charitidis, Carbon 37 (1999) 765.

[17] V. Kouznetsov, K. Macak, J.M. Schneider, U. Helmersson, I. Petrov, Surf. Coat. Technol. 122 (1999) 290.

[18] U. Helmersson, M. Lattemann, J. Bohlmark, A.P. Ehiasarian, J.T. Gudmundsson, Thin Solid Films 513 (2006) 1.

[19] K. Sarakinos, J. Alami, and S. Konstantinidis, Surf. Coat. Technol 204 (2010) 1661.

[20] J.T. Gudmundsson, J. Alami, U. Helmersson, Surf. Coat. Technol. 161 (2002) 249.

[21] J. Bohlmark, J. Alami, C. Christou, A.P. Ehiasarian, U. Helmersson, J. Vac. Sci. Technol. A 23 (2005) 18. 
[22] J. Alami, P. Eklund, J.M. Andersson, M. Lattemann, E. Wallin, J. Bohlmark, P. Persson, U. Helmersson, Thin Solid Films 515 (2007) 3434.

[23] S. Konstantinidis, A. Hemberg, J.P. Dauchot, M. Hecq, J. Vac. Sci. Technol. B 25 (2007) L19.

[24] J. Alami, K. Sarakinos, F. Uslu, M. Wuttig, J. Phys. D: Appl. Phys. 42 (2009) 015304.

[25] A.P. Ehiasarian, P.E. Hovsepian, L. Hultman, U. Helmersson, Thin Solid Films 457 (2004) 270.

[26] J. Alami, P. Eklund, J. Emmerlich, O. Wilhelmsson, U. Jansson, H. Högberg, L. Hultman, U. Helmersson, Thin Solid Films 515 (2006) 1731.

[27] S. Konstantinidis, J.P. Dauchot, M. Hecq, Thin Solid Films 515 (2006) 1182.

[28] J. Alami, K. Sarakinos, F. Uslu, C. Klever, J. Dukwen, M. Wuttig, J. Phys. D: Appl. Phys. 42 (2009) 115204.

[29] J. Paulitsch, P.H. Mayrhofer, C. Mitterer, W.-D. Münz, M. Schenkel, Society of Vacuum Coaters 50th Annual Technical Conference Proceedings, Louisville, KY, 2007, p. 150.

[30] V. Sittinger, F. Ruske, W. Werner, C. Jacobs, B. Szyszka, D.J. Christie, Thin Solid Films 516 (2007) 5847.

[31] K. Sarakinos, J. Wördenweber, F. Uslu, P. Schulz, J. Alami, and M. Wuttig, Surf. Coat. Technol. 202 (2008) 2323.

[32] S.P. Bugaev, V.G. Podkovyrov, K.V. Oskomov, S.V. Smaykina, N.S. Sochugov, Thin Solid Films 389 (2001) 16. 
[33] B.M. DeKoven, P.R. Ward, R.E. Weis, D.J. Christie, R.A. Scholl, W.D.

Sproul, F. Tomasel, A. Anders, Society of Vacuum Coaters 46th Annual

Technical Conference Proceedings, San Francisco, CA, 2003, p. 158.

[34] P. Patsalas, S. Logothetidis, P.C. Kelires, Diam. Relat. Mater. 14 (2005)

1241.

[35] L. Calliarri, Diam. Relat. Mater.14 (2005) 1232.

[36] P. Patsalas, M. Handrea, S. Logothetidis, M. Gioti, S. Kennou, W. Kautek, Diam. Relat. Mater.10 (2001) 960.

[37] J.C. Lascovich, S. Scaglione, Appl. Surf. Sci. 78 (1994) 17.

[38] K. Sarakinos, J. Alami, J. Dukwen, J. Wördenweber, M. Wuttig, J. Phys. D:

Appl. Phys. 41 (2008) 215301.

[39] M. Samuelsson, D. Lundin, J. Jensen, M. A. Raadu, J. T. Gudmundsson, U. Helmersson, Surf. Coat. Technol. 15 (2010) 591.

[40] W. Lotz, Z. Physik 232 (1970) 101.

[41] V. I. Merkulov, D. H. Lowndes, G. E. Jellison, A. A. Puretzky, D. B.

Geohegan Appl. Phys. Lett. 73 (1998) 2591.

[42] D.R. McKenzie, D. Muller, B.A. Pailthorpe, Phys. Rev. Lett. 67 (1991) 773.

[43] A.C. Ferrari, A. Libassi, B.K. Tanner, V. Stolojan, J. Yuan, L.M. Brown, S.E.

Rodil, B. Kleinsorge, J. Robertson, Phys. Rev. B 62 (2000) 11089.

[44] P.J. Fallon, V.S. Veerasamy, C.A. Davis, J. Robertson, G.A.J. Amaratunga,

W.I. Milne, J. Koskinen, Phys. Rev. B48 (1993) 4777.

[45] P. Patsalas, Thin Solid Films 519 (2011) 3990. 
[46] R. Kleber, K. Jung, H. Ehrhardt, I. Muhling, K. Breuer, H. Metz, F. Engelke, Thin Solid Films 205 (1991) 274.

[47] M. Weiler, S. Sattel, T. Giessen, K. Jung, H. Ehrhardt, V.S. Veerasamy, J. Robertson, Phys. Rev. B53 (1996) 1594. 


\section{Figure captions}

Fig. 1. Time-averaged ion energy distribution functions of (a) $\mathrm{Ar}^{+}$, (b) $\mathrm{C}^{+}$and (c) $\mathrm{H}_{2} \mathrm{O}^{+}$species in HiPIMS (solid line) and dcMS (dashed line) discharges as recorded by a mass-energy analyzer. The data presented here were recorded from a HiPIMS discharge operating at a frequency of $250 \mathrm{~Hz}$. The scale of the $\mathrm{Y}$ axis is logarithmic and the distance between to subsequent ticks represents an order of magnitude.

Fig. 2. Relative intensity fractions of characteristic ionized species in (a) dcMS and HiPIMS discharges at pulsing frequencies of (b) $1 \mathrm{kHz}$ and (c) $250 \mathrm{~Hz}$ as recorded by a mass-energy analyzer. The total integrated intensity is provided for each case. In addition, for the HiPIMS discharges the peak target power achieved at each pulsing frequency is also given.

Fig. 3. Fraction of $\mathrm{sp}^{3}$ hybridized bonds in films deposited by dcMS (full squares) and HiPIMS at pulsing frequencies of $1 \mathrm{kHz}$ (full circles) and $250 \mathrm{~Hz}$ (full triangles) as a function of the bias voltage applied on the substrate. Values of $\mathrm{sp}^{3}$ fractions measured in amorphous carbon films grown by rfMS (Ref. [16], hollow squares), FCA (Ref. [42], hollow circles), and PLD (Ref. [41], hollow triangles) as a function of the substrate bias voltage (dc voltage for Ref. [16]) or the incident ion energy (Refs. [41] and [42]) are reproduced for reference. 
Fig. 4. Mass density of films deposited by dcMS (filled squares) and HiPIMS at pulsing frequencies of $1000 \mathrm{~Hz}$ (full circles) and $250 \mathrm{~Hz}$ (full triangles) as a function of the bias voltage applied on the substrate. Values of films densities in amorphous carbon films grown by PLD (Ref. [12], hollow squares) are reproduced for reference.

Fig. 5. Mass density of films deposited by dcMS (half-full squares) and HiPIMS at pulsing frequencies of $1 \mathrm{kHz}$ (half-full circles) and $250 \mathrm{~Hz}$ (half-full triangles) as a function of $\mathrm{sp}^{3}$ bond fraction. Data from non-hydrogenated (full symbols) and hydrogenated (hollow symbols) amorphous carbon films (a-C and a-C:H, respectively) reported in the literature are also reproduced for reference. 
Figures

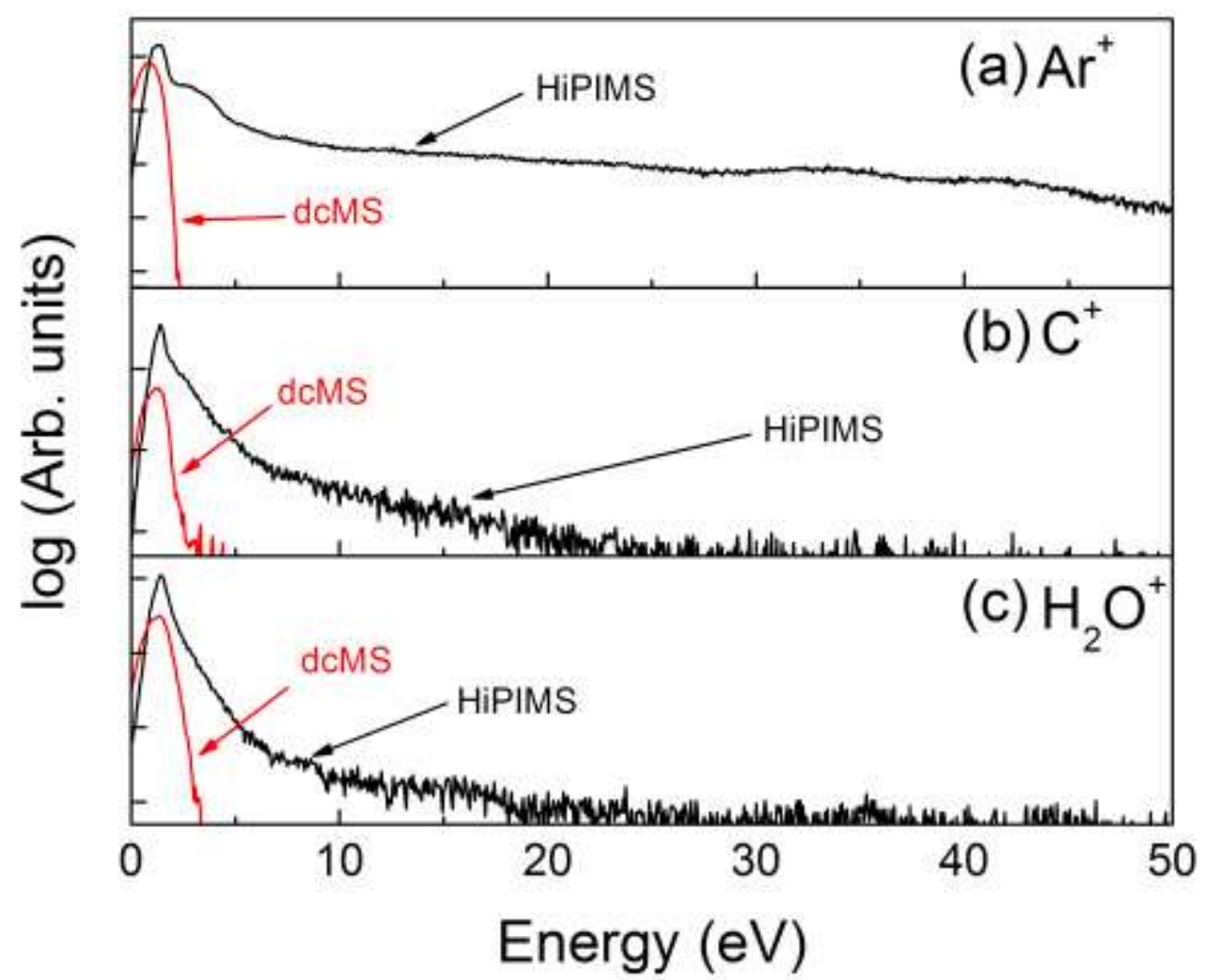

FIG. 1 


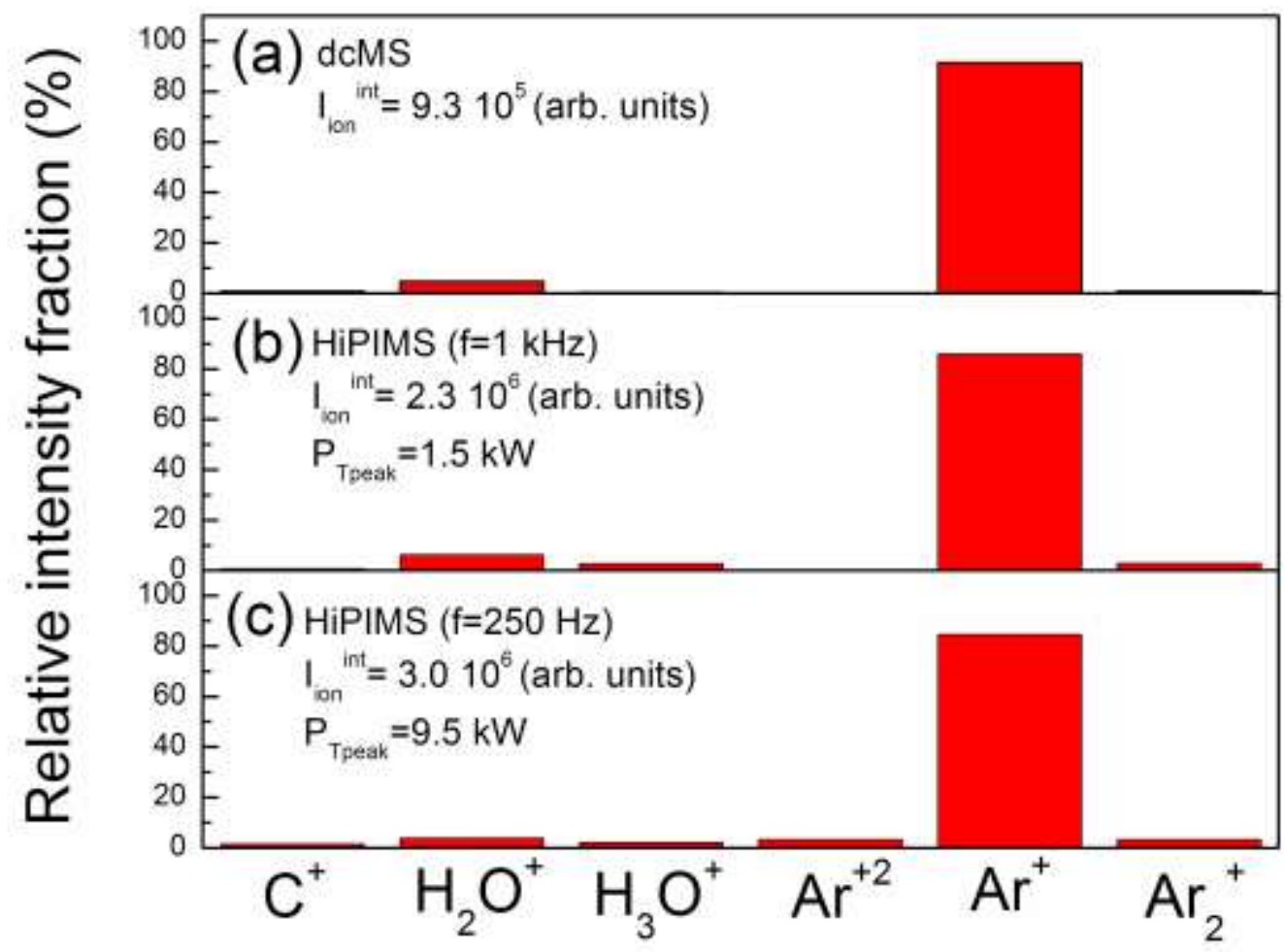

FIG. 2 


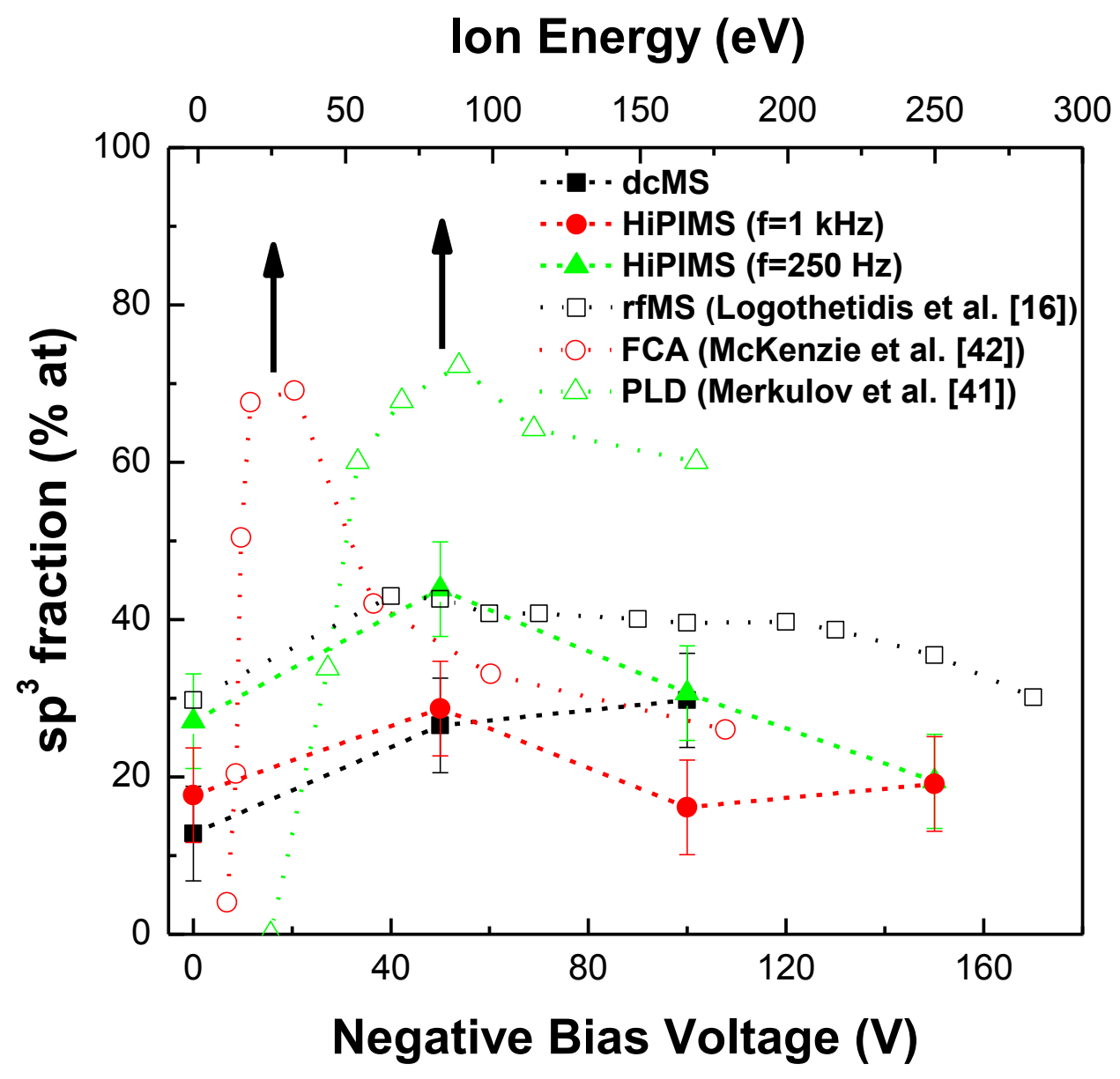

FIG. 3. 


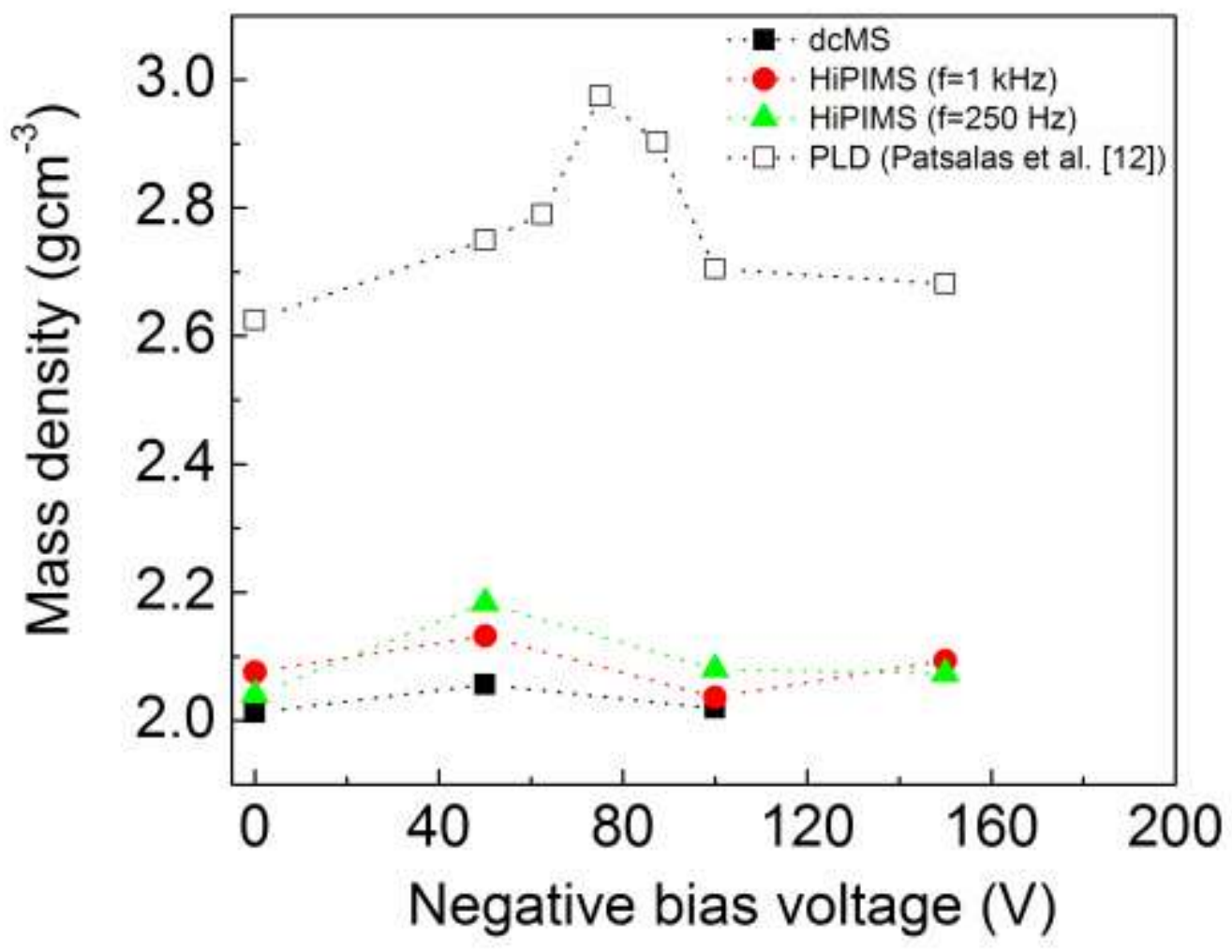

FIG. 4 


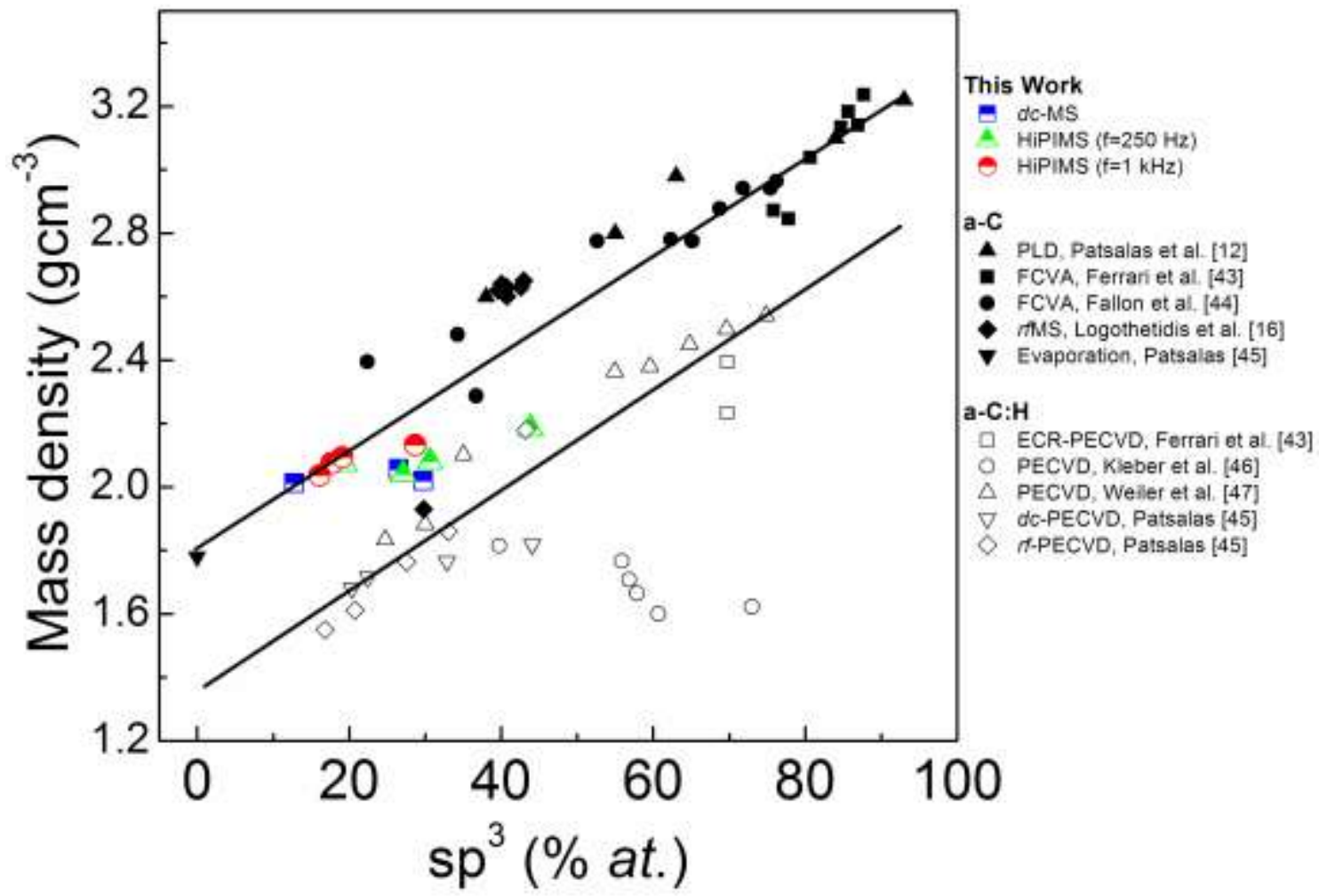

FIG. 5 